Cultures \& Conflits

08 | hiver 1992

Les conflits après la bipolarité

\title{
Les conflits post bipolaires : dynamiques et caractéristiques
}

Didier Bigo

\section{(2) OpenEdition}

1 Journals

\section{Édition électronique}

URL : http://journals.openedition.org/conflits/517

DOI : 10.4000/conflits. 517

ISSN : $1777-5345$

Éditeur :

CCLS - Centre d'études sur les conflits lilberté et sécurité, L'Harmattan

\section{Édition imprimée}

Date de publication : 6 décembre 1992

ISSN : 1157-996X

Référence électronique

Didier Bigo, "Les conflits post bipolaires : dynamiques et caractéristiques », Cultures \& Conflits [En ligne], 08 | hiver 1992, mis en ligne le 07 janvier 2003, consulté le 30 mars 2021. URL : http:// journals.openedition.org/conflits/517 ; DOI : https://doi.org/10.4000/conflits.517

Ce document a été généré automatiquement le 30 mars 2021.

Creative Commons License 


\title{
Les conflits post bipolaires : dynamiques et caractéristiques
}

\author{
Didier Bigo
}

1 Quelles sont les conditions dans lesquelles dorénavant ces conflits se développent? Que se passe-t-il maintenant que l'affrontement Est/Ouest a quasiment disparu et que la bipolarité n'est plus qu'un souvenir? Telle est la question qui court en filigrane dans les différents articles composant ce numéro ${ }^{1}$.

2 Zaki Laidi dans "penser l'après guerre froide" ouvre ce numéro en proposant une grille de lecture interprétative qui, par bien des aspects, renouvelle les problématiques existantes et soulève de nouvelles questions, tout à fait salutaires pour un meilleur entendement des situations contemporaines. Christian Lechervy à propos du Cambodge, Roland Marchal à propos de la corne de l'Afrique, Philippe Chapleau pour l'Afrique du Sud, Georges Couffignal pour l'Amérique Centrale et Christophe Jaffrelot sur les émeutes d'Ayodhia en Inde retrouvent souvent des accents identiques pour décrire les phénomènes conflictuels qu'ils retracent en détail en donnant des informations inédites. On y voit un découplage fréquent entre les conflits et la scène internationale, une autonomisation des acteurs locaux, le renforcement de la pression des acteurs militarisés sur les populations civiles désarmées qu'ils contrôlent, et l'usage de stratégies instrumentalistes où le recours à la violence armée pour des fins politiques ou d'appropriation privée de biens matériels devient extrêmement banal... nombre d'éléments que nous avions évoqué lors de la table ronde de notre premier numéro sur les "systèmes de guerre"2.

3 Entrelacé avec cette dimension très décentralisée, on voit émerger une autre ligne de force, à savoir la dimension transnationale où les réseaux d'acteurs transcendent les frontières étatiques et structurent autrement que par le jeu des alliances entre Etats l'évolution des formes conflictuelles. Roland Marchal, Christophe Jaffrelot, Rémy Leveau nous en donnent des illustrations significatives. Rémy Leveau en particulier montre comment le poids des populations immigrées ou de deuxième génération pèse de plus en plus sur les rapports politiques étatiques entre les Etats européens et ceux du Maghreb. Ces rapports directs qui créent des connexions intersociétales et 
modifient le jeu des Etats se retrouvent aussi dans la superbe analyse de Guy Nicolas sur les enjeux sacrificiels qui peuvent structurer les comportements de nombre d'acteurs minoritaires et sur leurs stratégies d'accès au "marché humanitaire", marché qui semble être le succédané maladroit de l'équilibre bipolaire. James Rosenau, quant à lui, dans un texte très stimulant et volontiers provocateur à l'égard de nos amis juristes, rappelle l'intérêt et les limites des réformes constitutionnelles dans un monde soumis simultanément à des dynamiques centralisatrices et décentralisatrices qui multiplient les tensions politiques et mettent à mal la cohérence du cadre étatique. Avec d'autres formules et dans un autre style, James Rosenau analyse ainsi comme Zaki Laidi les dynamiques intégratrices et désintégratrices qui entraînent le découplage du sens et de la puissance, et qui mettent à mal la prétention de l'Etat à monopoliser la force légitime dans l'ordre social interne et dans les relations internationales. Ne serait-ce pas alors cette faiblesse de l'Etat, bien au delà des régimes communistes, qui expliquerait alors l'augmentation en nombre et en intensité des conflits ouverts que nous connaissons depuis quelques années?

Les Dynamiques à l'oeuvre

4 Ce sont ces nouveaux paramètres qu'il peut être intéressant de relever. Il existerait d'un côté une dynamique d'intégration dont les vecteurs centraux seraient l'économie de marché capitaliste, le système de communication et les media audio-visuels, le système de transport en particulier aérien, et de l'autre une dynamique de décentralisation dont les vecteurs seraient l'augmentation par l'éducation des capacités analytiques des individus, leur nouveau rapport plus fonctionnaliste à l'obéissance, leur attachement à des structures d'opportunité politique plus proches d'eux que l'Etat, ce qui favoriserait le localisme, le particularisme, les micro-nationalismes et plus généralement des constructions identitaires porteuses de sens, refusant explicitement ou implicitement le primat du politique étatico-national. Se superposerait aussi à ces deux dimensions, celle plus spécifique des mouvements de solidarité transnationaux, liée aux mouvements de populations migrantes et aux rapports interculturels entretenus par ces acteurs. Cette dernière pertuberait aussi bien les tendances à l'homogénéisation et à la globalisation que les tendances à de stricts replis identitaires sur le local Ces trois dynamiques (décentralisatrice, globalisatrice et transnationale) jouent simultanément et peuvent expliquer l'évolution récente des conflits. Malheureusement nombre d'analyses n'en prennent qu'une seule en considération et s'enferment dans des visions réductrices du monde post bipolaire en terme d'unipolarité ou de désordre mondial.

5 La dynamique d'intégration ou de globalisation pousserait au dépassement des frontières étatiques et de leur mode de régulation, les englobant dans une logique toujours plus mondialiste. Cette dynamique de mondialisation rendrait plus ou moins obsolète les combats idéologiques et pousserait à la conversion de fait à la "démocratie de marché" de tous les régimes politiques, même lorsqu'ils prétendent le contraire. Il y aurait une sorte de "temps mondial", de "main stream" qui orienterait sans déterminer totalement les trajectoires historiques des sociétés.

De là le renouveau des thèses historicistes et développementalistes qui ne voient que cette dynamique à l'oeuvre et qui conclut un peu vite à la fin de l'Histoire, au parcours obligé pour les pays d'Europe centrale et orientale ainsi que pour les pays du Tiers Monde du chemin et des valeurs occidentales : économie de marché, élections libres, pluralisme politique. Hors de cette voie, point de salut $!^{3}$ 
7 D'autres s'émeuvent de cette confusion entre mondialisation et ethnocentrisme occidental. Ils réfutent cette vision téléologique de l'Histoire qui place les sociétés sur une échelle temporelle où l'on pourrait désigner qui est en avance et qui est en retard. Si personne ne semble réellement contester les effets de la mondialisation par l'économie, rien ne dit que sur le plan des valeurs démocratiques, il en soit de même. On ne voit guère les structures sociales qui porteraient à l'universalisation du pluralisme politique et des valeurs que l'Occident met sous le terme de démocratie. L'escroquerie du terme "démocratie de marché" serait patente. Elle serait un stratagème des puissances occidentales pour imposer leurs valeurs en même temps que leur économie. Les formes de la conditionnalité des prêts financiers au pays en voie de développement en serait un signe. Malgré ces critiques, les auteurs restent fascinés par cette seule dynamique d'intégration et pour eux la fin de la bipolarité signifie un monde unipolaire pour ne pas dire monopolaire où les Etats-Unis imposeraient leur domination, sinon via leur économie, du moins via leur système de valeur, leur langue, leur monnaie mondiale et leur capacités d'action militaire, soudainement unique au monde. Pour eux, le globe risque donc de devenir plus homogène mais ce n'est pas l'effet d'un mécanisme, c'est celui d'une volonté stratégique d'un acteur dominant à laquelle, après tout, on peut s'opposer et qui va peut être provoquer le chaos en voulant unifier le monde sous sa tutelle ${ }^{4}$.

8 Le paradoxe de ces discours, c'est que leur opposition manichéenne Bien/Mal, Structure/Volonté cache mal tous leurs présupposés communs et leur vision mutilante $\mathrm{du}$ monde post-bipolaire. Ne comprenant pas les effets des dynamiques de décentralisation et de transnationalité, qui sont à l'oeuvre pourtant depuis bien longtemps, ils sont incapables de penser le monde post-bipolaire autrement que comme une bipolarité soudain instable et déséquilibrée. Ils continuent d'utiliser les mêmes catégories d'analyse, les mêmes outils méthodologiques en les appliquant simplement à ce qui fut longtemps un cas d'école des théories bipolaires : l'horizon de la puissance impériale.

9 Concernant l'analyse des conflits, le premier courant neo Hegelien, ne veut voir dans les conflits actuels que la prolongation temporaire des séquelles de la bipolarité et des effets d'ajustement transitoire où il est finalement logique qu'une conflictualité ouverte éclate comme prémice à un ordre international globalement plus homogène. Développé surtout dans les premiers temps des accords Reagan-Gorbatchev où la fin de la guerre froide leur semblait logiquement devoir entrainer la fin des conflits "périphériques", leur discours continue de réapparaitre à chaque plan de paix (Amérique centrale, Afrique Australe, Afghanistan...) même si, à chaque fois il semble contredit sur le terrain. ${ }^{5}$ Leur reste alors l'espoir et les "visions" à long terme où le bruit des batailles s'estompe pour qui sait voir au loin.

10 Le second courant y voit à l'inverse les effets de la volonté impérialiste dominante, maintenant que le verrou de l'équilibre de la dissuasion entre les "deux" grands a sauté. Le recours à la guerre, aux interventions armées se substituerait à la dissuasion, les petites guerres chaudes (là où les intérêts l'exigent) à la grande guerre froide.

11 Les dynamiques de décentralisation posent plus de problème à l'analyse. Certains, on l'a vu, les négligent totalement et n'y voient que désordre, chaos, irrationnel ou nouveau vocable pour résumer ces thèmes : "libanisation". D'autres leur accordent au contraire une extrême importance mais ne leur donnent pas forcément les mêmes origines. Face à la dimension de l'économique et de la communication, on trouverait le 
"culturel", voire la "nature humaine". Les cultures reprendraient le dessus. Elles seraient les soubassements longtemps négligés du politique et de l'économique. L'exemple des pays d'Europe centrale et orientale, de la Russie, et même de l'ex Allemagne de L'Est montrerait à l'envie que les vieilles lignes de clivage entre les peuples slaves et les autres, entre Eglise d'Occident et Eglise d'Orient... seraient les facteurs déterminants de la vie politique. Le communisme ne serait qu'un épiphénomène qui aurait "gelé" un temps, grâce à sa force coercitive, les vieilles dynamiques culturelles et nationales ${ }^{6}$. Ce culturalisme différentialiste où la nation est essentialiste, où les cadres de vie sont prédéterminés et où l'histoire ne retient que les affrontements pour l'hégémonie entre peuples, races ou ethnies a sans doute profondément déconsidéré aux yeux des autres analystes la prise en compte de cette dynamique de décentralisation. Celle-ci n'est d'ailleurs plus une dynamique mais une constante transhistorique pour ces auteurs. Heureusement pour nombre de personnes le culturel est trop important pour le laisser aux culturalistes ${ }^{7}$. On ne comprend alors les dynamiques nationalitaires qu'en les analysant en terme de stratégies d'acteurs, où des "entrepreneurs politiques" mobilisent des groupes en fabriquant, à partir des significations imaginaires sociales majeures de la société, de nouveaux arrangements entre concepts tirés du "stock cognitif" de la société donnée, importés d'autres sociétés, ou même inventés. Loin d'une image fixiste de la culture, on a alors une dynamique qui met en jeu des stratégies diversifiées, concurrentes, antagonistes d'acteurs politiques sur un même espace et dont rien ne permet de prédire à l'avance qui l'emportera. La nation, l'ethnicité, le religieux se construisent ${ }^{8}$. Ils se font et se défont à chaque instant dans l'imaginaire politique et leur durée est à la fois bien réelle dans ces lignes de force, dans ses mises en forme et bien illusoire dans son contenu. Ce dernier n'étant souvent que reconstruction a posteriori par des groupes d'intellectuels autorisés qui naturaliseront l'arbitraire des constructions du moment en les rattachant à des mythes fondateurs et tout particulièrement à ceux qui s'originent dans le sacrificiel. C'est donc au niveau de l'acteur qu'il faut se situer pour comprendre ces dynamiques ; mais quel acteur? Si Zaki Laidi, James Rosenau, Christophe Jaffrelot, Guy Nicolas, Rémy Leveau... sont tous sensibles à ces dynamiques, et si, en même temps, ils sont tous très critiques à l'égard de ces visions culturalistes de l'Histoire, il n' y a plus forcément accord sur l' acteur à prendre en compte et sur les raisons profondes de ces dynamiques. Zaki Laidi évoque la problématique du "sens" mais n'identifie guère ces agents. Joue-t-elle comme la puissance au niveau des Etats, ce que suggère l'idée d'un découplage entre sens et puissance et son exemplification par le militaire, ou provientelle d'autres acteurs? Comment penser le "sens" au niveau international sans tellement simplifier qu'on prend le risque de le conceptualiser sous le signe de l'univocité ou même du primat hiérarchique? Celui-ci ne semble guère pouvoir se rapporter à la puissance. En posant ce type d'équation où sens et puissance serait éléments comparables afin d'analyser leur couplage ou leur découplage, ne risque-t-on pas d'assigner au sens, "un" sens et renouer avec une certaine téléologie qu'une approche plus sensible aux trajectoires multiples du politique dans chaque groupement humain et a fortiori à l'échelon international permettrait d'éviter? James Rosenau quant à lui contourne la difficulté de l'acteur collectif à laquelle Zaki Laidi s'affrontait. Pour lui les modifications du "sens" qui résultent avant tout des dynamiques de décentralisation sont à chercher dans la révolution des compétences individuelles. Partout avec les progrès de l'éducation les individus seraient plus aptes à défendre leurs intérêts, leurs valeurs propres (qui ne sont pas forcément celles de l'Occident), et 
seraient moins prêts à se soumettre aux autorités politiques par la simple loi de l'habitude. Celles-ci devraient maintenant montrer leurs capacités fonctionnelles. Séduisante, cette thèse "morphogénétique" où le même paramètre de départ prend des valeurs particulières selon le contexte, pose néanmoins problème. D'une certaine manière c'est toute la sociologie de l'action collective qui est court circuitée par le racourci sémantique du "paramètre micro-macro". A aucun moment Rosenau n'explique comment émerge un intérêt collectif, comment jouent les structures organisationnelles, quel est leur rapport à l'Etat... Sans doute prisonnier du jeu métaphorique qu'il institue entre l'analyse des relations internationales et les théories de l'auto-organisation, il multiplie les analogies en les faisant passer pour des explications. De plus on peut être sceptique sur ce "progrès" de l'éducation, sur cette "révolution des compétences" qu'il met en avant. Ne retrouve-t-on pas là un certain optimisme matiné d'évolutionnisme caractéristique d'une certaine pensée américaine? C'est sans doute à travers des études de terrain plus systématiques comme celles qu'engagent ici Rémy Leveau, Christophe Jaffrelot, Roland Marchal, Christian Lechervy , Georges Couffignal que l'on pourra mieux analyser sur le plan théorique ces dynamiques de décentralisation et les acteurs collectifs qui en sont les porteurs ${ }^{9}$.

Rémy Leveau met particulièrement en évidence dans son texte les dynamiques transnationales et la manière dont elles perturbent les jeux étatiques classiques. Ces dynamiques transnationales créent des liens au delà des frontières qui contredisent l'image de la fragmentation, de la décentralisation. Des courrants d'idées, de personnes circulent dans des espaces importants et créent des réseaux qui s'insinuent au coeur des Etats. Mais il montre aussi que ces dynamiques bien réelles ne se confondent pas avec celles de la globalisation. Ainsi par exemple ces tendances à la globalisation, sur le plan des représentations idéologiques fabriquent plus des fantasmes - le discours sur l'invasion homogénéise les jeunes beurs français, l'immigration maghrébine et les populations du maghreb - qu'elles ne sont capables de rendre compte d'une réalité qui, si elle dépasse bien les cadres nationaux ne va pas nécessairement dans le sens d'une globalisation. Guy Nicolas propose aussi plusieurs pistes de recherche qui semblent prometteuses en terme d'analyse d'interactions entre acteurs collectifs minoritaires, Etats et nouvelle structure partielle du jeu international, ce qu'il appelle le marché humanitaire. Cette troisième dynamique brouille les dichotomies, un peu facile, entre global et local, centralisé et décentralisé, homogéne et hétérogéne, intégrateur et fragmentaire. Nous avions proposé un modèle interprétatif des contestations populaires qui la prenne en considération dans le numéro sur les émeutes urbaines ${ }^{10}$. Peut-être peut-on en prolonger les hypothèses pour mieux expliquer d'autres formes de conflits.

Caractéristiques des conflits post-bipolaires

13 Les conflits post-bipolaires ne peuvent donc être lus sous l'angle unique des dynamiques de globalisation ou sous l'angle de la permanence culturelle. Ils ne peuvent pas être lus non plus comme la simple résultante de l'interaction globalisation fragmentation. Ils sont au minimum le produit de l'entrecroisement des dynamiques de centralisation, de décentralisation et de transnationalisation. Leur complexité, au sens fort du terme, repose sur cette "triade".

De plus localement certaines dynamiques peuvent produire, "loin de l'équilibre", des effets contraire à ceux qui en résultent généralement. Ainsi, la dynamique de centralisation, globalisation peut avoir des effets fortement hétérogènes selon les 
contextes locaux et créer elles-mêmes des conditions de fragmentation ou de clôture. Les logiques économiques peuvent par exemple pousser les gouvernant à abandonner la dimension étatique nationale mais pour privilégier un espace qu'ils espèrent plus maitrisables politiquement face au marché (échelon européen, grand marché américain...). De même la dynamique de décentralisation n'est pas forcément perturbatrice et génératrice de conflit, elle peut certes générer des fragmentations identitaires mais aussi des recompositions plus souples et plus adaptées à l'hybridation des répertoires contemporains : un certain mode de vie des jeunes dans les cités en France (langage spécifique, rapport à la musique...) en sont un signe. Enfin la dynamique transnationale souvent analysée à travers les vecteurs de l'économie informelle, du sociétal, de la valorisation du "bas" et d'une idéologie du "small is beautiful" peut aussi provoquer des phénomènes avec lesquels il faut compter: déterritorialisation de la violence par des organisations clandestines ou des gouvernants, rôle des connexions entre mafias, spécialisation de certains entrepreneurs comme des intermédiaires dans les trafics d'Etats... ${ }^{11}$. Il n'y aura donc pas, n'en déplaise à nos gouvernants et à leurs conseillers des systèmes prédictifs fiables pour analyser les conflits. Ces dynamiques, même une fois repérées sont trop instables ou trop créatrices pour pouvoir être extrapolées.

15 Et pourtant, contrairement à une idée reçue les conflits post-bipolaires ne sont pas plus complexes qu'auparavant. Ils ne sont pas non plus véritablement neufs et leur profusion tient plus à un changement de statut de la violence qu'à une période chaotique de l'ordre mondial.

16 Ils ne sont pas plus complexes dans la mesure où, au contraire, la bipolarité (que ce soit en période de guerre froide ou de détente) surajoutait à tout conflit local les exigences $\mathrm{du}$ jeu stratégique entre les deux grands et ce, surtout après la théorisation du linkage dans les années 70. Ce qu'on appela le globalisme et qui régna longtemps sur la stratégie est mort en même temps que l'effondrement soviétique, laissant côté occidental des orphelins à la recherche d'un nouvel ennemi global (le Sud, l'immigration, les mafias...) ${ }^{12}$

17 . Le localisme lui a survécu. Il continue de déterminer les modes de pensée des stratéges, au moins français et américains. Pour nombre d'entre eux, avec la fin de la stabilité bipolaire, nous sommes entrés dans une ère de "turbulences". En effet selon eux, la bipolarité contenait soi-disant les conflits périphériques en bloquant des logiques d'escalade, alors que maintenat ce frein n'existe plus. Les conflits seraient donc plus nombreux, plus meurtriers, nécessitant plus d'interventions armées pour les calmer... et ils tiendraient en dernière analyse au déchainement des passions guerrières maintenant que la raison stratégique n'est plus là pour les tempérer. Les peuples du Tiers Monde et pire les peuples d'Europe centrale et orientale se laisseraient aller à leurs "haines"... Outre le mépris implicite qu'ils entretiennent à l'égard des autres populations du monde via leur distinction entre les vrais conflits (raison stratégique) et les carnages guerriers (les passions), n'est-il pas nécessaire de rappeler que la bipolarité a aussi souvent attisé des conflits et $n$ ' a pas servi que de frein aux conflits comme les transferts d'armements le prouvent abondamment ${ }^{13}$

18 . La disparition de la bipolarité n'est donc pas forcément objet d'une augmentation du nombre et de l'intensité des conflits. Mal à l'aise avec l'étude polémologique et la sociologie des conflits, la vision stratégique (du fait de sa hauteur?) lorsqu'elle est confrontée à des analyses locales et ne peut plus se réfugier derrière les mots creux de 
stratégie de contournement ou de stratégies indirectes, donne alors la main au psychologisme (des peuples et non plus de "l'adversaire") en redonnant au mythe de l'instinct agressif de nouveaux jours de gloire. Matinée de culturalisme essentialiste, elle peut déboucher alors sur un racisme mal masqué par la fausse désolation devant l'abominable.

La simplification d'un des niveaux de la conflictualité restructure l'ensemble des enchevêtrements conflictuels et donne aux dynamiques de décentralisation un poids plus significatif. La sociologie des acteurs collectifs (militarisés et désarmés) apparait comme encore plus nécessaire à l'analyse. Elle permet d'éviter ce court-circuit du "stratégico-irrationnel" que nous voyons se développer à longueur de colonnes dans les journaux. Il faut suivre les stratégies rationnelles des acteurs militarisés et leur rapport à l'adversaire ainsi qu'aux populations sous contrôle. Il faut comprendre comment des "systèmes de guerre" se mettent en place et se perpétuent. Il faut voir leur fonctionnalité et leur intérêt à l'intérieur du champ politique lorsque celui-ci continue d'exister. Tâche qui nécessite d'aller sur le terrain, d'avoir des connaissances d'anthropologie, d'histoire des sociétés et de sociologie beaucoup plus que d'histoire militaire et de stratégie. Ne doutons pas que les résistances corporatistes à ce changement d'expertise dureront un certain temps.

A ce niveau d'ailleurs de la sociologie de la conflictualité, la permanence l'emporte sur la rupture. Seule l'illusion ethnocentrique européenne peut faire oublier le nombre de conflits qui ont eu lieu durant la bipolarité. Nombre des conflits actuels sont des conflits qui existaient déjà dans la période précédente et si un phénomène central a touché l'évolution des formes conflictuelles depuis les années 80, c'est celui de la perte de contrôle des populations et des frontières par les Etats des pays du Tiers Monde sous la poussée de nouveaux acteurs collectifs militarisés ( guerillas traditionnelles mais aussi autonomisation de secteurs coercitifs ou groupes liés à des réseaux de drogue). C'est donc dans le contexte global de la transnationalisation ou de la transfrontalièrité qu'il est nécessaire d'analyser l'évolution actuelle des conflits. Enfin s'il y a bien de "nouveaux conflits" avec ce qu'on a appelé le "retour du nationalisme", il ne faudrait pas oublier que cette augmentation de la violence expressive est à mettre en parallèle avec la gigantesque diminution de la violence coercitive dans de nombreuses zones du monde, ce qui nous renvoie une nouvelle fois à la pertinence de l'Etat comme seule structure d'opportunité politique. Certes celui-ci essaie de trouver de nouvelles légitimations pour son ordre coercitif mais il est de plus en plus soumis à la montée d'autres acteurs collectifs qui refusent sa prétention au monopole de la violence et il sera obligé, si ce n'est déjà fait, de transiger avec eux plutôt que de se raccrocher à sa puissance régalienne.

\section{NOTES}

1. Contrairement à notre habitude, ce numéro réunit des articles qui ont été commandé indépendamment les uns des autres. Il ne faut donc pas y chercher une cohérence de 
problématique et une articulation précise autour d'un thème construit et discuté par les différents auteurs. Il a néanmoins émergé un problème auquel sont confrontés les auteurs qui analysent les conflits contemporains. : celui de la pertinence du cadre conceptuel hérité de la bipolarité

2. Cultures \& Conflits $n^{\circ} 1:$ La prolongation des conflits

3. Nombre de programmes proposés par les gouvernements occidentaux aux pays d'Europe centrale et orientale s'enracinent dans ces présupposés dont Francis Fukuyama s'est fait, parfois à son corps défendant, le défenseur le plus populaire. Beaucoup d'hommes politiques, de milieux diplomatiques, de philosophes médiatiques et de journalistes éditorialistes semblent avoir partagé cette analyse.

4. Voir les derniers tenants de la sociologie de la dépendance, les courants pacifistes.... Parmi bien d'autres ouvrages signalons celui d' Alain Joxe : l'Amérique mercenaire Paris Stock 1992.

5. Pour une approche critique de ces visions voir Didier Bigo Daniel Hermant "La métamorphose des conflits" in Etudes Polémologiques n50 2/89

6. Nombre d'analystes des pays de l'Est se sont reconvertis à cette hypothèse du "frigidaire".

7. $C^{\prime}$ est une des motivations de notre revue.

8. Voir tout particulièrement les différents ouvrages de Bertrand Badie

9. C'est aussi tout l'intérêt du livre de Zaki Laidi sur l'ordre mondial relâché par rapport à certaines hypothèses beaucoup plus générales et par là pas assez sensibles aux différences de trajectoires et à la diversité mondiale.

10. Voir Didier Bigo : "les jeux du politique et de la transnationalité" in Cultures \& Conflits $n^{\circ} 5$ Emeutes urbaines le retour du politique

11. Voir Cultures \& Conflits $n^{\circ} 4:$ Réseaux internationaux de violence

12. Voir Cultures \& Conflits $n^{\circ} 2$ Menaces du Sud : images et réalités, Cultures \& Conflits $\mathrm{n}^{\circ} 3$ mafia drogue et politique

13. Pour une analyse plus serrée des faiblesses du globalisme, et des visions géostratégiques, voir Didier Bigo : "les interprétations des années 1989/1990 enjeux et problématiques" in Approche polémologique FEDN, 1991

\section{RÉSUMÉS}

Quelles sont les conditions dans lesquelles dorénavant ces conflits se développent? Que se passet-il maintenant que l'affrontement Est/Ouest a quasiment disparu et que la bipolarité n'est plus qu'un souvenir ? Telle est la question qui court en filigrane dans les différents articles composant ce numéro.

This issue continues the discussion begun by the first issue, concerning "the prolongation of conflicts", at a more structural level. How will armed conflicts develop in the future ? What is happening now that the East-West confrontation and bipolarity have disappeared ? The logic of the actors, the reasons for prolongation induced by the socialisation of war and the difficulties for armed people to accept returning to a civilian way of life are anlaysed, as well as the new context after bipolarity. The development of recent conflicts can be analysed via three dynamic 
movements : globalisation, fragmentation, transnationalisation. Globalisation and fragmentation are analysed as two opposite dynamics. Some analysts only see globalisation and speak of unipolarity, others only see fragmentation and speak of " chaos ", " southern threat ", or " clash of civilisations ". New theories propose combining globalisation and fragmentation in order to understand the " turbulences " of the present World, but they lack a clear distinction between globalisation, fragmentation and transnationalisation. This last dynamic, is however, not the result of the first two but follows its own logic.

\section{INDEX}

Mots-clés : Relations Internationales, sociologie des conflits (polémologie)

Index chronologique : post-guerre froide

\section{AUTEUR}

\section{DIDIER BIGO}

Maître de conférences des Universités à l'Institut d'Etudes Politiques de Paris, rédacteur en Chef de Cultures \& Conflits 